\title{
SILVICULTURA DE Acacia mearnsii NO SUL DO BRASIL: BIOMASSA E NUTRIENTES
}

\author{
SILVICULTURE OF Acacia mearnsii IN SOUTHERN BRAZIL: BIOMASS AND NUTRIENTS
}

\author{
Grasiele Dick', Mauro Valdir Schumacher ${ }^{1}$ \\ ${ }^{1}$ Universidade Federal de Santa Maria, Santa Maria, Rio Grande do Sul, Brasil - grasidick@hotmail.com \\ \&mauro.schumacher@ufsm.br
}

\section{RESUMO}

\begin{abstract}
A silvicultura de Acacia mearnsii é uma importante atividade econômica no Rio Grande do Sul e a colheita da biomassa deve visar sempre a sustentabilidade nutricional do sítio. O objetivo deste trabalho é caracterizar o estoque de nutrientes contido na biomassa e a exportação em função de diferentes intensidades de colheita de uma plantação de Acacia mearnsii, na região central do Rio Grande do Sul, Brasil. Nove árvores foram fracionadas em folhas, galhos, casca da madeira e madeira do tronco, de onde foram determinados os pesos secos e teores de nutrientes. A produção total de biomassa é de $174,67 \mathrm{Mg} \mathrm{ha}^{-1}$, onde $72,8 \%$ corresponde à madeira do tronco e $12,5 \%$ à casca da madeira. Houve maior acúmulo dos nutrientes $\mathrm{N}, \mathrm{K}, \mathrm{Ca}$ e Fe e, o sistema de colheita onde há retirada da casca da madeira e madeira do tronco, preconizado na acacicultura, remove mais de $50 \%$ das quantidades de nutrientes acumuladas na biomassa, o que torna indispensável a manutenção dos resíduos (folhas e galhos) sobre o solo.
\end{abstract}

PALAVRAS-CHAVE: Acácia-negra, Nutrição florestal, Manejo de resíduos.

\section{ABSTRACT}

Acacia mearnsii silviculture is an important economic activity in Rio Grande do Sul state and biomass harvesting should always aim at the nutritional sustainability of the site. The objective of this work is to characterize the nutrient stock contained in the biomass and the export in function of different harvest intensities of a Acacia mearnsii plantation, in the central region of Rio Grande do Sul state, Brazil. Nine trees were fractionated in leaves, branches, stembark and stemwood, from where were determined the dry weights and contents of nutrients. The total biomass production was $174.67 \mathrm{Mg} \mathrm{ha}^{-1}$, where $72.8 \%$ corresponds to the stemwood and $12.5 \%$ to the stembark. There was a greater accumulation of $\mathrm{N}, \mathrm{K}, \mathrm{Ca}$ and Fe nutrients, and the harvesting system where there is a removal of the stembark and stemwood, recommended in acaciculture, removes more than $50 \%$ of the nutrient amounts accumulated in the biomass, which maintenance of the waste (leaves and branches) on the soil.

KEYWORDS: Black wattle, Forest nutrition, Residue management. 


\section{INTRODUÇÃO}

A prática da silvicultura vem se expandindo nos últimos anos em todo o mundo, visto que no ano de 2005 havia 105 milhões de hectares com plantações, que passaram para 291 milhões em 2015 (FAO, 2005; 2016). Dentre as principais espécies cultivadas, junto aos gêneros Pinus e Eucalyptus, se destaca a Acacia, sendo que, até o ano de 2014, a área com acacicultura no Brasil era de 160.872 ha (IBÁ, 2015) e, o estado do Rio Grande do Sul detém $64 \%$ destas plantações, especialmente com a espécie Acacia mearnsii De Wild. (AGEFLOR, 2015). A extração da casca para produção de tanino, fabricação de papel e celulose, aglomerados e energia a partir da madeira são as principais finalidades do cultivo desta espécie, realizada em sua maioria, por pequenos produtores rurais no sul do Rio Grande do Sul (CALDEIRA et al., 2006).

Acacia mearnsii, conhecida popularmente como acácia-negra, é uma árvore leguminosa de uso versátil, de valor comercial agregado na madeira e casca, apresenta bom crescimento em sítios inadequados para a agricultura e propicia melhorias na fertilidade do solo (DUNLOP \& MACLENNAN, 2002). Estas modificações edáficas são promovidas pela capacidade de fixação biológica de nitrogênio, além da ciclagem de nutrientes via serapilheira, o que viabiliza a recomendação do plantio de Acacia mearnsii para recuperação de áreas em pastagens degradadas (COSTA et al., 2014). Além disto, plantações da espécie atuam na fixação do carbono atmosférico, podendo armazenar mais de $60,0 \mathrm{Mg} \mathrm{ha}^{-1} \mathrm{na}$ biomassa das árvores, aos sete anos de idade (SANQUETTA et al., 2014).

Em função da fixação biológica, Acacia mearnsii é utilizada em consórcio com espécies agrícolas e arbóreas, em sua maioria Eucalyptus (VIERA et al., 2013a; BAUHUS et al., 2000; KHANNA, 1997). Estas combinações promovem aumento da produtividade das plantações, por exemplo, Bauhus et al. (2000) verificaram que, em um sistema com $50 \%$ acácia e $50 \%$ eucalipto, o volume de madeira é de $47 \mathrm{~m}^{3} \mathrm{ha}^{-1}$, já no monocultivo de eucalipto a produtividade é de $21 \mathrm{~m}^{3} \mathrm{ha}^{-1}$. Neste mesmo sistema, Forrester et al. (2004) e Bauhus et al. (2004) também verificam maior produção total de biomassa de eucalipto e acácia quando comparado ao monocultivo. Tais estudos evidenciam a função ecológica e importância silvicultural da espécie, no entanto, ainda há carência de informações sobre as melhores combinações e potencial desses sistemas para a produção sustentável (VIERA et al., 2013b).
Prezar pela sustentabilidade ecológica de plantações de Acacia mearnsii é fundamental em um cenário de expansão da silvicultura, que é baseada em monocultivos conduzidos, geralmente, em solos de baixa fertilidade. Para tanto, predições quanto ao manejo de resíduos da colheita a permanecerem sobre o piso florestal, são estratégias que visam a redução da exaustão nutricional e manutenção da capacidade de produção dos povoamentos nos diferentes tipos de solos (CALDEIRA et al., 2002).

Para o manejo silvicultural, as avaliações do estoque de nutrientes acumulados na biomassa de Acacia mearnsii são necessárias e os conhecimentos sobre a distribuição dos nutrientes nos componentes das árvores desta espécie são fundamentais para estudos de nutrição, ciclagem e exportação dos nutrientes (CALDEIRA et al., 2006). Ressalta-se que, após a colheita destas plantações, a sustentabilidade nutricional poderá ser comprometida, pois além da madeira, também há total retirada da casca, o que implica na remoção de grandes quantidades de nutrientes, dentro de um período de tempo relativamente curto de rotação da espécie (TOIT, 2002).

O objetivo deste trabalho é caracterizar o estoque de nutrientes contido na biomassa e a exportação em função de diferentes intensidades de colheita de uma plantação de Acacia mearnsii, cultivada na região central do Rio Grande do Sul, Brasil.

\section{MATERIAL E MÉTODOS}

\section{Caracterização ecológica do sítio}

O estudo foi realizado em uma plantação de 5,0 hectares de Acacia mearnsii, com oito anos de idade, na Unidade de Pesquisa Florestal da Fundação Estadual de Pesquisa Agropecuária (coordenadas geográficas centrais $29^{\circ} 40^{\prime} 07^{\prime \prime}$ S; 5354' 47" O). Para o plantio das mudas de Acacia mearnsii, realizado em outubro de 2005 , utilizouse o espaçamento de 3,0 m entre linhas e 1,3 m entre plantas (densidade inicial de 2.500 árvores ha-1). O solo foi preparado em área nova, através do sistema mecanizado com grade rotativa, em profundidade média de $30 \mathrm{~cm}$ na linha de plantio e, na entrelinha a faixa de solo revolvido foi de $1,0 \mathrm{~m}$ de largura, sem nenhuma aplicação de fertilizante. Realizou-se replantio após 40 dias, mantendo a taxa de sobrevivência das plantas superior a $90 \%$.

A área está localizada no município de Santa Maria, em altitude de $113 \mathrm{~m}$, compreendendo a região fisiográfica da depressão central do estado do Rio Grande do Sul, Brasil. O clima da região é subtropical úmido, do 
tipo Cfa (Köppen) e a temperatura média anual é de $19,4^{\circ} \mathrm{C}$, com média dos meses mais quentes superior a $30^{\circ} \mathrm{C}$ (dezembro e janeiro) e dos meses mais frios entre 13 e $18{ }^{\circ} \mathrm{C}$ (julho e agosto). A precipitação média anual é de $1.581,4 \mathrm{~mm}$ (ALVARES et al., 2014).

O solo da área é do tipo Argissolo Vermelho Distrófico arênico (EMBRAPA, 2013) e a caracterização da fertilidade (Tabela 1) foi realizada antes do plantio e aos oito anos de idade, através da coleta e análise química de amostras retiradas de seis trincheiras, em profundidades de 0 a 10 $\mathrm{cm}, 10$ a $20 \mathrm{~cm}, 20$ a $30 \mathrm{~cm}$ e 30 a $40 \mathrm{~cm}$. A matéria orgânica (MO) e o fósforo $(\mathrm{P})$ disponível (extrator Melich1) foram determinados por espectrofotometria, o potássio (K) por fotometria de chama e o cálcio (Ca) e magnésio $(\mathrm{Mg})$ por espectrofotometria de absorção atômica (TEDESCO et al., 1995). Para estimativa da concentração de $\mathrm{N}$ no solo, foi assumido o valor de $10 \%$ do teor de matéria orgânica (GONÇALVES et al., 2001).

Tabela 1. Fertilidade do solo antes e após oito anos de cultivo de Acacia mearnsii na região central do Rio Grande do Sul.

\begin{tabular}{|c|c|c|c|c|c|c|c|c|c|}
\hline \multirow{2}{*}{\multicolumn{2}{|c|}{ Prof. (cm) }} & \multicolumn{2}{|c|}{0 a 10} & \multicolumn{2}{|c|}{10 a 20} & \multicolumn{2}{|c|}{20 a 30} & \multicolumn{2}{|c|}{30 a 40} \\
\hline & & S1 & $S 2$ & S1 & S2 & S1 & S2 & S1 & $S 2$ \\
\hline MO & \multirow{2}{*}{$\frac{\vec{b}}{\vec{b}}$} & 12,0 & 14,0 & 8,0 & 8,0 & 8,0 & 9,0 & 8,0 & 10,0 \\
\hline $\mathrm{N}$ & & 1,2 & 1,4 & 0,8 & 0,8 & 0,8 & 0,9 & 0,8 & 1,0 \\
\hline $\begin{array}{c}\mathrm{pH} \\
\mathrm{H}_{2} \mathrm{O}\end{array}$ & , & 4,7 & 4,7 & 4,7 & 5,0 & 4,6 & 4,8 & 4,6 & 4,6 \\
\hline $\mathrm{Ca}$ & \multirow{2}{*}{$\begin{array}{l}\text { है } \\
\text { है } \\
\overline{0} \\
\text { है }\end{array}$} & 0,7 & 1,4 & 0,4 & 1,4 & 0,3 & 1,6 & 0,2 & 0,7 \\
\hline $\mathrm{Mg}$ & & 0,3 & 0,6 & 0,1 & 0,8 & 0,1 & 1,1 & 0,1 & 0,5 \\
\hline K & \multirow{2}{*}{ 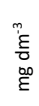 } & 52,1 & 50,5 & 40,5 & 40,5 & 37,2 & 45,0 & 35,7 & 39,1 \\
\hline$P$ & & 2,5 & 3,5 & 2,1 & 2,3 & 2,4 & 2,0 & 2,5 & 2,0 \\
\hline
\end{tabular}

Prof. = profundidade de coleta; $\mathrm{S} 1$ = análise de solo antes do plantio; $\mathrm{e}$ S2 = análise de solo aos oito anos da plantação.

Segundo a Comissão de Química e Fertilidade do Solo - RS/SC (CQFS, 2016), o teor de matéria orgânica no solo é baixo $\left(\leq 25,0 \mathrm{~g} \mathrm{~kg}^{-1}\right)$, os valores de $\mathrm{pH}$ e $\mathrm{P}$ são muito baixos ( $\leq 5,0$ e $\leq 7,0$, respectivamente), o Ca é baixo $(\leq 2,0$ $\left.\mathrm{cmol}_{\mathrm{c}} \mathrm{dm}^{-3}\right)$ e $\mathrm{Mg}$ varia de médio a alto $\left(0,6>1,0 \mathrm{cmol}_{\mathrm{c}}\right.$ $\left.\mathrm{dm}^{-3}\right)$.

\section{Produção de biomassa, acúmulo e exportação de nutrientes}

Previamente à coleta de biomassa, foram instaladas três parcelas de $15 \mathrm{~m}$ x $20 \mathrm{~m}$, de forma aleatória, onde se realizou a mensuração, com uso de fita diamétrica, de todos os diâmetros à altura do peito (DAP) das árvores, resultando em um DAP médio de $15,6 \mathrm{~cm}$, com intervalo de classe variando de $9,8 \mathrm{~cm}$ até $22,1 \mathrm{~cm}$. Aos oito anos da plantação, a densidade de árvores por hectare é de, aproximadamente, 2.250 plantas.

De cada parcela foram abatidas três árvores de DAP médio, seccionadas ao nível do solo e fracionadas nos componentes: folhas, galhos (mortos e vivos), casca da madeira e madeira do tronco (diâmetro $\geq 6,0 \mathrm{~cm}$; ponteira $<6,0 \mathrm{~cm}$ inclusa na fração galhos). Foi obtido o peso úmido de cada fração com auxílio de balança de gancho (precisão $\pm 150 \mathrm{~g}$ ) e para estimativa da biomassa total, multiplicou-se a biomassa das três árvores de DAP médio pelo número de árvores da parcela, e posteriormente, extrapolou-se para hectare $\left(\mathrm{Mg} \mathrm{ha}^{-1}\right)$.

De cada fração, coletaram-se amostras que foram processadas em laboratório, passando por secagem em estufa de circulação forçada de ar, a $70{ }^{\circ} \mathrm{C}$, por 72 horas, até peso constante, para determinação do peso seco. Em seguida, as amostras foram moídas e encaminhadas para a determinação analítica das concentrações de macro e micronutrientes, segundo metodologias de Tedesco et al. (1995). O teor de nitrogênio ( $N$ ) foi determinado pelo método Kjeldahl, via digestão sulfúrica $\left(\mathrm{H}_{2} \mathrm{SO}_{4}+\mathrm{H}_{2} \mathrm{O}_{2}\right)$, o boro (B) via digestão seca e para os nutrientes cálcio (Ca), magnésio $(\mathrm{Mg})$, cobre $(\mathrm{Cu})$, ferro $(\mathrm{Fe})$, manganês $(\mathrm{Mn})$, zinco $(Z n)$, potássio $(K)$, fósforo $(P)$ e enxofre $(S)$, a digestão utilizada foi a nítrica-perclórica $\left(\mathrm{HNO}_{3}+\mathrm{HClO}_{4}\right.$, na proporção 3:1), determinados por espectrofotometria.

O cálculo do estoque de nutrientes nos compartimentos da biomassa de Acacia mearnsii foi efetuado por meio do produto da concentração média de nutrientes pela biomassa. Já para a estimativa do cenário de exportação de nutrientes da plantação, foram consideradas três intensidades de colheita: 1 의 remoção somente da madeira do tronco (M); 2 ㅇ) retirada de madeira do tronco + casca da madeira $(\mathrm{M}+\mathrm{C}) ; 3$ 의 colheita total da biomassa acima do solo (BT).

\section{Análise estatística}

Para detecção de diferenças entre médias das concentrações dos nutrientes nos diferentes componentes da biomassa, utilizou-se o teste Tukey, com probabilidade de $5 \%$ de erro, no delineamento inteiramente casualizado. 


\section{RESULTADOS E DISCUSSÃO}

\section{Biomassa e estoque de nutrientes}

A biomassa de Acacia mearnsii aos oito anos de idade é de $174,67 \mathrm{Mg} \mathrm{ha}^{-1}$, sendo que $127,12 \mathrm{Mg} \mathrm{ha}^{-1}$ está acumulada na madeira do tronco (72,8\%), 21,91 $\mathrm{Mg} \mathrm{ha}^{-1}$ $(12,5 \%)$ correspondem à casca da madeira, $18,44 \mathrm{Mg} \mathrm{ha}^{-1}$ $(10,6 \%)$ aos galhos e as folhas representam 7,20 $\mathrm{Mg} \mathrm{ha}^{-1}$ $(4,1 \%)$.

A prioridade para alocação de biomassa nas árvores segue a tendência de madeira do tronco > casca da madeira > galhos > folhas, resultados diferentes dos estudos de Caldeira et al. (2011), Sanquetta et al. (2014) e Barrichelo et al. (2005), onde os autores constam maior produção de galhos em detrimento à casca da madeira, para plantações de 4, 7 e 8 anos, respectivamente. Estudos verificam que a quantidade de casca da madeira que a espécie produz, quando cultivada em Argissolo e também em solos rasos na região sul do Brasil, pode variar de 0,15 $\mathrm{Mg} \mathrm{ha}^{-1}$ aos seis meses de idade das árvores (VIERA \& SCHUMACHER, 2011), passando para 7,0 Mg ha-1 aos quatro anos e até mais de $14,0 \mathrm{Mg} \mathrm{ha}^{-1}$ aos sete anos da plantação (SANQUETTA et al., 2014).

A biomassa total estimada neste estudo se assemelha aos resultados encontrados por Sanquetta et al. (2014) e Barichello et al. (2005) para plantações aos sete anos de idade (134,8 $\mathrm{Mg} \mathrm{ha}^{-1}$ e 115,6 $\mathrm{Mg} \mathrm{ha}^{-1}$, respectivamente). No entanto, em áreas com plantações da espécie em solos arenosos, ácidos e distróficos na Austrália, Bauhus et al. (2004) constatam biomassa total acima do solo de $60,0 \mathrm{Mg} \mathrm{ha}^{-1}$ e Forrester et al. (2004) quantificam 72,0 Mg $\mathrm{ha}^{-1}$, ambos estudos realizados aos nove anos de idade das árvores.

No presente estudo se observa que, além da grande produção de casca da madeira, o componente folhas também contribui para o elevado acúmulo de biomassa, pois as maiores proporções são constatadas, geralmente, quando as plantações são mais jovens, onde 5,9 $\mathrm{Mg} \mathrm{ha}^{-1}$ de folhas foram quantificadas aos 1,5 anos (VIERA \& SCHUMACHER, 2011), 7,2 $\mathrm{Mg} \mathrm{ha}^{-1}$ aos 2 anos (CALDEIRA et al., 2001) e 5,6 Mg ha ${ }^{-1}$ aos 3 anos da espécie (SANQUETTA et al., 2014).

A elevada produção de biomassa de Acacia mearnsii, especialmente a casca da madeira, é característica de qualidade almejadas nos monocultivos da espécie. Os resultados deste estudo podem estar atrelados a diversos fatores, tais como o plantio em Argissolo (RACHWAL et al., 2008), baixa mortalidade, o adequado o preparo de solo que condicionou melhorias físicas e maior profundidade ao desenvolvimento radicular das plantas, aliada à boa drenagem, que são algumas das exigências ao cultivo da espécie, que é tolerante à baixa fertilidade (DUNLOP \& MACLENNAN, 2002).

As frações das árvores apresentaram distintas composições químicas, e as concentrações dos nutrientes diferem especialmente nas folhas, onde há maiores concentrações de N, P, S, B, Cu, Fe e Mn. Macronutrientes como $\mathrm{K}, \mathrm{Ca}$ e $\mathrm{Mg}$ não diferem entre os componentes folhas, galhos e casca da madeira e na madeira do tronco há menor concentração de todos os nutrientes (Tabela 2).

Tabela 2. Concentrações de nutrientes na biomassa de Acacia mearnsii.

\begin{tabular}{|c|c|c|c|c|c|c|}
\hline & $\mathbf{N}$ & $\mathbf{P}$ & $\mathbf{K}$ & $\mathrm{Ca}$ & $\mathbf{M g}$ & $S$ \\
\hline & \multicolumn{6}{|c|}{$\mathrm{g} \mathrm{kg}^{-1}$} \\
\hline $\mathrm{F}$ & $31,08 a^{*}$ & $1,06 a$ & $9,53 \mathrm{a}$ & $7,31 \mathrm{a}$ & $1,93 \mathrm{a}$ & $1,17 \mathrm{a}$ \\
\hline G & $11,97 \mathrm{~b}$ & $0,36 \mathrm{~b}$ & $8,10 \mathrm{a}$ & $6,58 \mathrm{a}$ & $1,60 \mathrm{a}$ & $0,44 \mathrm{~b}$ \\
\hline C & $12,53 \mathrm{~b}$ & $0,29 b c$ & $7,84 \mathrm{a}$ & 9,08 a & $1,33 \mathrm{a}$ & $0,33 b$ \\
\hline \multirow[t]{3}{*}{$M$} & $2,01 \mathrm{c}$ & $0,06 \mathrm{c}$ & $3,41 b$ & $0,86 \mathrm{~b}$ & $0,32 b$ & $0,20 \mathrm{~b}$ \\
\hline & B & $\mathrm{Cu}$ & $\mathrm{Fe}$ & & & $\mathrm{Zn}$ \\
\hline & \multicolumn{6}{|c|}{$\mathrm{mg} \mathrm{kg}^{-1}$} \\
\hline $\mathrm{F}$ & 29,50 a & $10,43 a$ & $151,39 a$ & 81 & & $15,00 \mathrm{a}$ \\
\hline G & $11,34 \mathrm{bc}$ & $4,54 \mathrm{~b}$ & $114,10 \mathrm{~b}$ & 21 & & $17,45 \mathrm{a}$ \\
\hline C & $14,48 \mathrm{~b}$ & $1,92 \mathrm{c}$ & $52,39 \mathrm{c}$ & 17 & & $10,23 \mathrm{~b}$ \\
\hline$M$ & $3,41 \mathrm{c}$ & $1,54 \mathrm{c}$ & $31,19 \mathrm{c}$ & 7, & & $10,11 b$ \\
\hline
\end{tabular}

$\mathrm{F}=$ folhas; $\mathrm{G}=$ galhos; $\mathrm{C}=$ casca do tronco; $\mathrm{M}=$ madeira do tronco; ${ }^{*}$ Médias seguidas pela mesma letra na vertical não diferem significativamente pelo teste de Tukey $5 \%$ de probabilidade.

Para os nutrientes acumulados na biomassa, em sua maioria mais concentrados nas folhas, a tendência de acúmulo nesta fração segue a ordem decrescente de $\mathrm{N}>$ $\mathrm{K}>\mathrm{Ca}>\mathrm{Mg}>\mathrm{S}>\mathrm{P}$, já para os micronutrientes está é de $\mathrm{Fe}>\mathrm{Mn}>\mathrm{B}>\mathrm{Zn}>\mathrm{Cu}$. Este mesmo padrão para os macronutrientes é observado por Caldeira et al. (2002; 2006) e Viera et al. (2013a), no entanto, para os micronutrientes as concentrações não seguem as mesmas tendências.

Nas folhas, onde há intensa atividade metabólica em função dos processos fotossintéticos (MARSCHNER, 2012), se concentram maiores teores dos nutrientes (exceto $\mathrm{Ca}$ e $\mathrm{Zn}$ ) em relação às demais frações da árvore, o que também é observado em outros estudos com a espécie (KHANNA 1997; CALDEIRA et al., 2002; 2006; VIERA et al., 2013a). Ressalta-se que, mais de $40 \%$ dos micronutrientes podem se concentrar nas folhas das árvores jovens de Acacia mearnsii (CALDEIRA et al., 2006) e em plantas de até seis meses, as concentrações foliares de $\mathrm{N}, \mathrm{P}, \mathrm{Mg}, \mathrm{B}, \mathrm{Mn}$ e $\mathrm{Zn}$ tendem a ser expressivamente 
maiores quando comparadas às plantações em idade de corte (VIERA et al., 2013a).

Muitos são os fatores que influenciam a composição mineral das folhas, desde fertilidade do solo, idade da planta, clima e também a mobilidade no floema ou remobilização do elemento. Por exemplo, o nitrogênio, absorvido através da fixação biológica, é o nutriente com mais concentrado nas folhas e, tanto quanto o fósforo e potássio, possui elevada mobilidade no floema. São minerais requeridos em maiores concentrações pela planta, pois participam de processos metabólicos, compõem estruturas de aminoácidos, proteínas e ácidos nucléicos (MARSCHNER, 2012). Já em relação ao Ca, nutriente de baixa mobilidade no floema, as maiores concentrações na fração casca da madeira se devem à função estrutural deste elemento, constituinte da lamela média de membrana celular (VIERA e SCHUMACHER, 2009).

Em relação às quantidades de nutrientes estocados na biomassa da plantação (Tabela 3), na madeira do tronco, que é o componente com maior biomassa alocada, há maior acúmulo de $\mathrm{K}, \mathrm{Mg}$, $\mathrm{S}$ e todos os micronutrientes. Os elementos $\mathrm{N}$ e Ca estão em maior quantidade na casca da madeira e $P$ nas folhas.

Tabela 3. Quantidade de nutrientes na biomassa de Acacia mearnsii.

\begin{tabular}{|c|c|c|c|c|c|c|}
\hline & $\mathbf{N}$ & $\mathbf{P}$ & $\mathbf{K}$ & $\mathrm{Ca}$ & $\mathrm{Mg}$ & $\mathbf{S}$ \\
\hline & \multicolumn{6}{|c|}{$\mathrm{kg} \mathrm{ha}^{-1}$} \\
\hline $\mathrm{F}$ & 222,02 & 7,57 & 65,36 & 51,59 & 13,68 & 7,92 \\
\hline G & 221,47 & 5,95 & 143,79 & 105,76 & 26,83 & 7,54 \\
\hline C & 276,42 & 6,23 & 165,22 & 195,16 & 28,38 & 7,05 \\
\hline \multirow[t]{3}{*}{ M } & 245,80 & 7,20 & 392,33 & 106,58 & 42,16 & 24,24 \\
\hline & B & & $\mathrm{Cu}$ & $\mathrm{Fe}$ & $M n$ & $\mathrm{Zn}$ \\
\hline & \multicolumn{6}{|c|}{$\mathrm{g} \mathrm{ha}^{-1}$} \\
\hline $\mathrm{F}$ & 224,19 & & 75,35 & $1.131,71$ & 567,19 & 109,85 \\
\hline G & 187,58 & & 77,98 & $2.051,96$ & 397,29 & 315,34 \\
\hline C & 320,48 & & 42,57 & $1.178,68$ & 412,19 & 217,10 \\
\hline$M$ & 448,77 & & 204,26 & $3.757,22$ & $1.046,21$ & $1.315,65$ \\
\hline
\end{tabular}

$\mathrm{Na}$ biomassa total, o macronutriente presente em maior quantidade é o $\mathrm{N}\left(965,71 \mathrm{~kg} \mathrm{ha}^{-1}\right)$, seguido de $\mathrm{K}$ $\left(766,70 \mathrm{~kg} \mathrm{ha}^{-1}\right), \mathrm{Ca}\left(459,09 \mathrm{~kg} \mathrm{ha}^{-1}\right), \mathrm{Mg}\left(111,05 \mathrm{~kg} \mathrm{ha}^{-1}\right), \mathrm{S}$ $\left(46,75 \mathrm{~kg} \mathrm{ha}^{-1}\right)$ e $\mathrm{P}\left(26,95 \mathrm{~kg} \mathrm{ha}^{-1}\right)$. Dos micronutrientes, o maior acúmulo é de Fe $\left(8.119,57 \mathrm{~g} \mathrm{ha}^{-1}\right)$, seguido de $\mathrm{Mn}$ $\left(2.422,88 \mathrm{~g} \mathrm{ha}^{-1}\right), \mathrm{Zn}\left(1.957,94 \mathrm{~g} \mathrm{ha}^{-1}\right), \mathrm{B}\left(1.181,02 \mathrm{~g} \mathrm{ha}^{-1}\right) \mathrm{e}$ $\mathrm{Cu}\left(400,16 \mathrm{~g} \mathrm{ha}^{-1}\right)$. As mesmas tendências de estoque, para todos os nutrientes, também são verificadas em plantios da espécie aos seis meses de idade (VIERA et al., 2013b). Cabe ressaltar que, à medida que aumenta o acúmulo de biomassa, há maior estoque de nutrientes na planta, especialmente na madeira do tronco (CALDEIRA et al., 2002; 2006; VIERA et al., 2013b).

Importante destacar que, a plantação de Acacia mearnsii estoca $128 \%, 1.050 \%$ e $54 \%$ a mais nitrogênio na biomassa das frações folhas + galhos, casca da madeira e madeira do tronco, respectivamente, em comparação a espécie Eucalyptus saligna aos 8,5 anos de idade, também cultivada na região da depressão central do Rio Grande do Sul, no mesmo tipo de solo (SANTANA et al., 2008). O nitrogênio é um nutriente indispensável na acacicultura, devendo ser adicionado via fertilização mineral para alcance de altas produtividades, pois muitos dos plantios desta espécie são realizados em solos distróficos e há elevado acúmulo do elemento na biomassa (SCHUMACHER et al., 2013).

\section{Exportação de nutrientes pela colheita da biomassa}

Dentre os possíveis sistemas considerando as intensidades de colheita das árvores da plantação de Acacia mearnsii (Figuras 1 e 2), aquele onde há remoção da biomassa total (BT) é o que mais exportaria nutrientes do sítio, sendo removidas as maiores quantidades, em ordem decrescente, de $\mathrm{N}>\mathrm{K}>\mathrm{Ca}>\mathrm{Mg}>\mathrm{S}>\mathrm{P}>\mathrm{Fe}>\mathrm{Mn}$ $>\mathrm{Zn}>\mathrm{B}>\mathrm{Zn}$. Este cenário causará grandes impactos na fertilidade do solo e provável requerimento de fertilização para uma nova rotação na área.

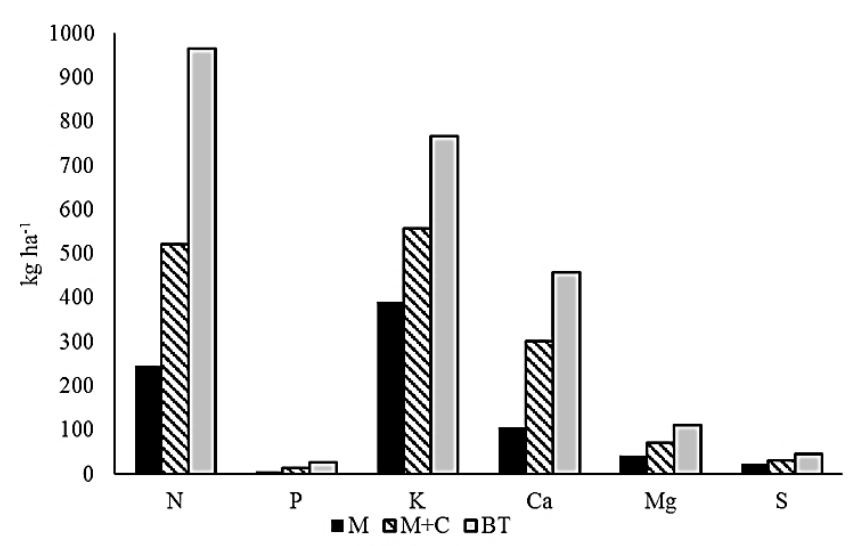

Figura 1. Exportação de macronutrientes $\left(\mathrm{kg} \mathrm{ha}^{-1}\right)$ em diferentes intensidades de colheita em plantação de Acacia mearnsii.

Em que: $M=$ remoção somente da madeira do tronco; $M+C=$ retirada de madeira do tronco + casca da madeira; $\mathrm{BT}=$ colheita total da biomassa acima do solo.

Ao simular a colheita de madeira com casca $(\mathrm{M}+\mathrm{C})$, verifica-se que ainda há exportação de mais de $50 \%$ da 
quantidade de cada nutriente. Já a retirada somente da madeira (M) propiciará a manutenção de 74\%, 73\%, 49\%, $77 \%$, 62\% e 76\%, dos macronutrientes N, P, K, Ca, Mg e S, respectivamente, ao passo que, neste sistema são mantidos no sítio $62 \%, 50 \%, 53 \%, 56 \%$ e $32 \%$ dos micronutrientes $\mathrm{B}, \mathrm{Cu}, \mathrm{Fe}, \mathrm{Mn}$ e $\mathrm{Zn}$, respectivamente.

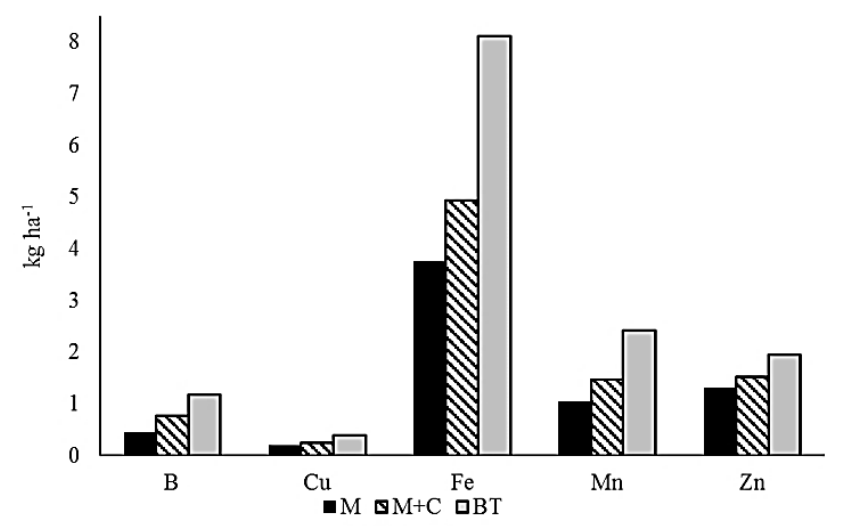

Figura 2. Exportação de micronutrientes $\left(\mathrm{kg} \mathrm{ha}^{-1}\right)$ em diferentes intensidades de colheita em plantação de Acacia mearnsii.

Em que: $\mathrm{M}=$ remoção somente da madeira do tronco; $\mathrm{M}+\mathrm{C}=$ retirada de madeira do tronco + casca da madeira; $\mathrm{BT}=$ colheita total da biomassa acima do solo.

Apesar da maior permanência de nutrientes no sítio, o sistema mais conservacionista, onde há somente a colheita de madeira do tronco, mantendo a casca da madeira junto aos demais resíduos da copa sobre o solo é pouco aplicado à acacicultura. De acordo com Caldeira et al. (2002), dentre os sistemas de exploração, o mais usual para a espécie, na idade de corte entre 7 e 9 anos de idade, é o que explora a madeira do tronco e casca da madeira até o diâmetro mínimo de $6,0 \mathrm{~cm}$, deixando a ponteira no campo, juntamente com galhos e folhas. Os autores afirmam ainda que, este sistema de colheita possibilita manter uma grande quantidade de resíduos sobre o solo e, consequentemente elevada quantidade de nutrientes, que serão liberados dessa biomassa após o processo de decomposição.

No entanto, contrariando o manejo conservacionista, muitos acacicultores optam pela queima dos resíduos para geração de energia, facilitar o preparo do solo para a nova rotação e também para manejar da praga Oncideres impluviata (besouro serrador-da-acácia-negra), de acordo com o que é recomendado pela Lei № 9482/1991 (RIO GRANDE DO SUL, 1991). Exceto para o controle sanitário, os resíduos da colheita devem ser mantidos sobre o solo, pois além de contribuir com a melhoria da fertilidade através da liberação gradativa de todos os nutrientes contidos nas folhas, galhos e ponteiras, também condicionarão melhorias nas propriedades físicas, por meio da redução de erosão e compactação, dentre outros impactos oriundos do preparo de solo para o próximo cultivo (TOIT, 2002).

\section{CONCLUSÕES}

Aos oito anos de idade, a plantação de Acacia mearnsii acumula grandes quantidades de nutrientes na madeira do tronco e casca do tronco, em função da elevada produção de biomassa. A exportação via colheita no sistema madeira do tronco + casca do tronco $(\mathrm{M}+\mathrm{C})$ implicará em remoção de mais de $50 \%$ dos nutrientes acumulados. Este impacto na fertilidade do solo é mitigado através da manutenção dos resíduos, compostos por folhas e galhos, sobre o solo. Desde que bem conduzido o manejo de resíduos pós-colheita, o plantio da espécie na região central do Rio Grande do Sul também deverá ser fomentado e recomendado, uma vez que, este é realizado em maiores escalas na região sul do estado.

\section{REFERÊNCIAS}

AGEFLOR - Associação Gaúcha de Empresas Florestais. A indústria de base florestal no RS: dados e fatos - Ano base 2014. AGEFLOR: Rio Grande do Sul, 2015.

ALVARES, C.A. et al. Köppen's climate classification map for Brazil. Meteorologische Zeitschrift, v.22, n.6, p.711-728, 2014.

BARICHELO, L.R. et al. Quantificação da biomassa de um povoamento de Acacia mearnsii De Wild. na região Sul do Brasil. Ciência Florestal, v.15, n.2, p.129-135, 2005.

BAUHUS, J.; et al. Aboveground and belowground interactions in mixed plantations of Eucalyptus globulus and Acacia mearnsii. Canadian Journal of Forest Research, v.30, p. 1886-1894, 2000.

BAUHUS, J. et al. Aboveground interactions and productivity in mixed-species plantations of Acacia mearnsii and Eucalyptus globulus. Canadian Journal of Forest Research, v.34, p.686-694, 2004.

CALDEIRA, M.V.W. et al. Quantificação da biomassa acima do solo de Acacia mearnsii De Wild., procedência Batemans Bay Austrália. Ciência Florestal, v.11, n.2, p.79-91, 2001.

CALDEIRA, M.V.W. et al. Exportação de nutrientes em função do tipo de exploração em um povoamento de Acacia mearnsii De Wild. Floresta e Ambiente, v.9, n.1, p.97-104, 2002.

CALDEIRA, M.V.W. et al. Conteúdo e exportação de micronutrientes em acácia-negra (Acacia mearnsii De Wild.) procedência Batemans Bay (Austrália), Revista Árvore, v.27, n.1, p.9-14, 2006.

CQFS - Comissão de Química e Fertilidade do Solo. Manual de adubação e calagem para os estados do Rio Grande do Sul e 
Santa Catarina. 2.ed. Porto Alegre, 2016.

COSTA, M.G. et al. Leguminosas arbóreas para recuperação de áreas degradadas com pastagem em Conceição de Macabu, Rio de Janeiro, Brasil. Scientia Forestalis, v.42, n.101, p.101-112, 2014.

DUNLOP, R.W.; MACLENNAN, L.A. Black Wattle: The South African Research Experience. ICFR, 2002.

EMBRAPA - Empresa Brasileira de Pesquisa Agropecuária. Sistema brasileiro de classificação de solos. 3.ed. Brasília: Embrapa Solos, 2013.

FAO - Food and agriculture organization of the United Nations. Global Forest Resources: Assessment update 2005. Roma: FAO, 2005.

FAO - Food and agriculture organization of the United Nations. Global Forest Resources Assessment 2015: How are the world's forests changing? Second edition. Roma: FAO, 2016.

FORRESTER, D.I. et al. Growth dynamics in a mixed-species plantation of Eucalyptus globulus and Acacia mearnsii. Forest Ecology and Management, v.193, p.81-95, 2004.

GONÇALVES, J.L.M. et al. Mineralização de nitrogênio em ecossistemas florestais naturais e implantados do estado de São Paulo. Revista Brasileira de Ciência do Solo, v.25, n.3, p.601616, 2001.

IBÁ - Indústria Brasileira de Árvores. Dados e estatísticas 2015. IBÁ, 2015.

KHANNA, P.K. Comparison of growth and nutrition of young monocultures and mixed stands of Eucalyptus globulus and Acacia meamsii. Forest Ecology and Management, v.94, p.105113, 1997.

MARSCHNER, P. Mineral nutrition of higher plants. 3.ed. New York: Academic Press; 2012.

RACHWAL, M.F.G. et al. A influência das características pedológicas na produtividade de acácia-negra (Acacia mearnsii), Butiá, RS. Pesquisa Florestal Brasileira, v.56, p.53-62, 2008.

RIO GRANDE DO SUL. Lei № 9482, de 24 de dezembro de 1991. Dispõe sobre o controle obrigatório do "serrador" da acácianegra e dá outras providências. 1991. Disponível em: http://ww1.sema.rs.gov.br/conteudo.asp?cod_menu=408\&cod_ conteudo $=7472$

SANQUETTA, C.R. et al. Estoques de biomassa e carbono em povoamentos de acácia negra em diferentes idades no Rio Grande do Sul. Scientia Forestalis, v.42, n.103, p.361-370, 2014.

SANTANA, R.C. et al. Alocação de nutrientes em plantios de eucalipto no Brasil. Revista Brasileira de Ciência do Solo, v.32, p.2723-2733, 2008.

SCHUMACHER, M.V. et al. Crescimento da acácia-negra em resposta a aplicação de nitrogênio, fósforo e potássio. Cerne, v.9, n.1, p.51-58, 2013.
TEDESCO, M.J. et al. Análise de solo, plantas e outros materiais. 2ed. Porto Alegre: UFRGS, 1995.

TOIT, B. Long-term ecological sustainability of wattle plantations. Black Wattle: The South African Research Experience. ICFR, 2002.

VIERA, M.; SCHUMACHER, M.V. Biomassa em povoamentos monoespecíficos e mistos de eucalipto e acácia-negra e do milho em sistema agrossilvicultural. Cerne, v.17, n.2, p.259-265, 2011.

VIERA, M.; SCHUMACHER, M.V. Concentração e retranslocação de nutrientes em acículas de Pinus taeda L. Ciência Florestal, v.29, n.4, p.375-382, 2009.

VIERA, M. et al. Teores de nutrientes em povoamentos monoespecíficos e mistos de Eucalyptus urograndis e Acacia mearnsii em sistema agrossilvicultural. Ciência Florestal, v.23, n.1, p.67-76, 2013a.

VIERA, M. et al. Estoque de nutrientes em consórcios de Eucalyptus urograndis, Acacia mearnsii e Zea mays. Ciência Florestal, v.23, n.3, p.317-327, 2013b. 